\title{
Bell-Plessett effect on harmonic evolution of spherical Rayleigh-Taylor instability in weakly nonlinear scheme for arbitrary Atwood numbers
}

\author{
Wanhai Liu, ${ }^{1,2}$ Changping $\mathrm{Yu}^{2,3, a)}$ Hongbin Jiang, ${ }^{1}$ and Xinliang $\mathrm{Li}^{2,3, a)}$ \\ ${ }^{1}$ Research Center of Computational Physics, Mianyang Normal University, Mianyang 621000, China \\ ${ }^{2}$ LHD, Institute of Mechanics, Chinese Academy of Sciences, Beijing 100190, China \\ ${ }^{3}$ School of Engineering Science, University of Chinese Academy of Sciences, Beijing 100049, China
}

(Received 25 August 2016; accepted 27 December 2016; published online 3 February 2017)

\begin{abstract}
Based on the harmonic analysis [Liu et al., Phys. Plasmas 22, 112112 (2015)], the analytical investigation on the harmonic evolution in Rayleigh-Taylor instability (RTI) at a spherical interface has been extended to the general case of arbitrary Atwood numbers by using the method of the formal perturbation up to the third order in a small parameter. Our results show that the radius of the initial interface [i.e., Bell-Plessett (BP) effect] dramatically influences the harmonic evolution for arbitrary Atwood numbers. When the initial radius approaches infinity compared against the initial perturbation wavelength, the amplitudes of the first four harmonics will recover those in planar RTI. The BP effect makes the amplitudes of the zeroth, second, and third harmonics increase faster for a larger Atwood number than smaller one. The BP effect reduces the third-order negative feedback to the fundamental mode for a smaller Atwood number, and strengthens it for a larger one. Hence, the BP effect helps the fundamental mode grow faster for a smaller Atwood number. Published by AIP Publishing. [http://dx.doi.org/10.1063/1.4973835]
\end{abstract}

\section{INTRODUCTION}

One of the most important factors, which can limit target performance in inertial confinement fusion (ICF), is an unstable growth of target nonuniformities leading to capsule disruption during the implosion, reducing a neutron yield, and Rayleigh-Taylor instability (RTI) is the most dangerous one. ${ }^{1-3}$ The RTI has been extensively investigated theoretically, ${ }^{4-14}$ experimentally, ${ }^{15,16}$ and numerically. ${ }^{17-20}$ Before the RTI enters a strong nonlinear stage, ${ }^{21-24}$ it will undergo a linear stage, and then a weakly nonlinear stage.

Generally, the RTI occurs on an interface separating a light fluid of density $\rho_{l}$ and another heavier one of density $\rho_{h}$ $\left(\rho_{l}<\rho_{h}\right)$ at cases: the light fluid supporting the heavier one in a gravity field $-g \boldsymbol{e}_{\boldsymbol{y}}$ where $g$ is acceleration or accelerating the heavier fluid. ${ }^{25,26}$ The density difference of the fluids on both sides of the interface is expressed as a normalized quantity, namely, Atwood number $A=\left(\rho_{h}-\rho_{l}\right) /\left(\rho_{h}+\rho_{l}\right)$. Under the above conditions, any small amplitude perturbation of the interface can stimulate the RTI. Assume that an initial perturbation is in the form $y=\eta(x, t=0)=\varepsilon \cos (k x)$ with $k \varepsilon \ll 1$ on an interface, in which $k=2 \pi / \lambda$ is wave number, $\lambda$ is perturbation wavelength, and $\varepsilon$ is a perturbation amplitude of the initial interface, then this interface will grow. Initially, the initial cosine modulation with a small amplitude grows exponentially in time $t, \eta_{L}=\varepsilon e^{\gamma t}$, where $\gamma=\sqrt{A k g}$ is the linear growth rate. ${ }^{25,26}$ When the perturbation amplitude is close to its wavelength, the second harmonic, third harmonic and so on are generated successively, and then the perturbation goes into the weakly nonlinear regime. ${ }^{12-14}$ In the third-order

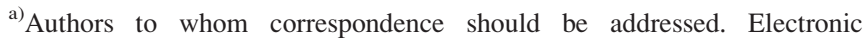
addresses: champion-yu@163.com and lixl@imech.ac.cn
}

weakly nonlinear theory, ${ }^{4-7}$ the interface position at time $t$ can be expressed as the form of $\eta(x, t)=\sum_{i=1}^{3} \eta_{i} \cos (i k x)$ with $\eta_{i}$ being the amplitude of the $i$ th harmonic

$$
\begin{gathered}
\eta_{1}=\eta_{L}-\frac{1}{16}\left(3 A^{2}+1\right) k^{2} \eta_{L}^{3} \\
\eta_{2}=-\frac{1}{2} A k \eta_{L}^{2} \\
\eta_{3}=\frac{1}{2}\left(A^{2}-\frac{1}{4}\right) k^{2} \eta_{L}^{3}
\end{gathered}
$$

From these three expressions, one finds that the growth of the fundamental mode is reduced by the nonlinear effect (i.e., thirdorder negative feedback to the fundamental mode) [see Eq. (1a)], for arbitrary Atwood numbers the amplitude of the second harmonic is always negative, showing the corresponding phase to be opposite to the initial cosine modulation's (anti-phase) [see Eq. (1b)] and the amplitude of the third harmonic can be positive or negative, depending on the Atwood number: when $A<1 / 2$, it is positive; otherwise, it is negative [see Eq. (1c)]. ${ }^{12-14}$

For the spherical RTI in incompressible viscous fluids, several investigations on linear stability analysis were carried out. The analysis ${ }^{27}$ in terms of spherical surface harmonics $Y_{n}$ of degree $n$ of the first kind was performed and the dispersion relation was obtained. Mikaelian ${ }^{20}$ studied the linear stability of arbitrary number $N$ of spherical concentric shells undergoing a radial implosion or explosion, by deriving the evolution equation for the perturbations on every interface, and for the $N=2$ case, obtained several analytical solutions just valid for class A and class B. The class-A solutions are for the specific $n A(n)$, an expression of mode number $n$ and Atwood number 
$A$ [see Eq. (28b) in Ref. 20], but arbitrary evolutional radius $R(t)$; the class-B solutions are the reverse: valid for arbitrary $n A(n)$, but only specific $R(t)$. The class-A solutions, respectively, for $n A(n)=-2$ and 0 , showed that the perturbation amplitude is related closely to the radius of the interface and the amplitude does not grow for the $R(t)$, and then a critical Atwood number is determined. The class-B solutions mainly discussed the four cases of the specific radial history $R(t)$. In the above works, they assumed that the growing perturbations, compared with the radius of the interface, are small, and a source or a sink exists at the origin to keep a constant density of the fluid inside of the spherical interface.

Based on the third-order weakly nonlinear theory in the Cartesian coordinate system, several works have been performed. Ref. 12 employed the method of the formal perturbation up to the tenth order in a small amplitude parameter to investigate the higher-order effect on nonlinear saturation amplitude of the fundamental mode. Ref. 13 gave a secondorder theory in a cylindrical coordinate system for arbitrary Atwood numbers to study the cylindrical effect on RTI, namely, the effect of the initial radius of the interface known as Bell-Plessett effect ${ }^{28,29}$ motivated by compression and geometrical convergence. As for the Bell-Plessett (BP) effect, its importance and relative investigations ${ }^{30-34}$ in RTI, the detailed introduction can be found in Ref. 14, in which the evolution of the first four harmonics in the spherical RTI is analytically investigated just for the case of $A=1$, without assuming a source or a sink to exist at the spherical center to maintain a constant density of the region inside of the spherical interface.

This work has been extended to the general case of arbitrary Atwood numbers including $A=1$. In other words, the evolution of the first four harmonics in the spherical RTI for irrotational, incompressible, and inviscid fluids with a discontinuous profile for arbitrary Atwood numbers is investigated analytically.

\section{THEORETICAL FRAMEWORK AND ANALYTICAL RESULTS}

This section plans to devote to the detailed description of the theoretical framework of this paper, and the explicit results of amplitudes of the first four harmonics are demonstrated.

In a spherical coordinate system $(r, \varphi, \theta)$, in which $r, \varphi$, and $\theta$ are, respectively, the radial coordinate, the angle measured down from the $z$-axis, and the azimuthal angle in the $x-y$ plane (here, the $x, y$, and $z$ are coordinates in the Cartesian system), there are two fluids with different densities separated by a spherical interface $r=r_{0}$. For some reasons, there always exist perturbations at the material interface. According to relations of the acceleration direction and fluid distribution, two cases can motivate the spherical RTI. ${ }^{13}$ The first means the acceleration pointing to the center of the spherical system and the heavy (light) fluid occupying the outer (inner) space of the spherical interface, and the other case is in complete antithesis to the first. Here, we focus mainly on the first case where the interface perturbation, for simplicity, just distributes in the direction of the $\theta$. In the following discussion, we shall denote the properties of the fluid outside the interface by the subscript $h$ and that inside the interface by the subscript $l$ unless otherwise stated. Assuming the two fluids in a gravitational field $-g \boldsymbol{e}_{r}$ to be irrotational, incompressible, and inviscid, the governing equations for this system are

$$
\begin{gathered}
\frac{\partial}{\partial r}\left(r^{2} \frac{\partial \phi_{i}}{\partial r}\right)+\frac{1}{\sin ^{2} \varphi} \frac{\partial^{2} \phi_{i}}{\partial \theta^{2}}=0 \quad \text { in two fluids } \\
\frac{\partial s}{\partial t}+\frac{1}{r^{2} \sin ^{2} \varphi} \frac{\partial s}{\partial \theta} \frac{\partial \phi_{l}}{\partial \theta}-\frac{\partial \phi_{l}}{\partial r}=0 \quad \text { at } \quad r=s(\theta, t), \\
\frac{\partial s}{\partial t}+\frac{1}{r^{2} \sin ^{2} \varphi} \frac{\partial s}{\partial \theta} \frac{\partial \phi_{h}}{\partial \theta}-\frac{\partial \phi_{h}}{\partial r}=0 \quad \text { at } \quad r=s(\theta, t), \\
(1-A)\left[\frac{\partial \phi_{l}}{\partial t}+\frac{1}{2}\left(\frac{\partial \phi_{l}}{\partial r}\right)^{2}+\frac{1}{2 r^{2} \sin ^{2} \varphi}\left(\frac{\partial \phi_{l}}{\partial \theta}\right)^{2}+g r\right] \\
-(1+A)\left[\frac{\partial \phi_{h}}{\partial t}+\frac{1}{2}\left(\frac{\partial \phi_{h}}{\partial r}\right)^{2}+\frac{1}{2 r^{2} \sin ^{2} \varphi}\left(\frac{\partial \phi_{h}}{\partial \theta}\right)^{2}+g r\right] \\
+f(t)=0 \quad \text { at } \quad r=s(\theta, t),
\end{gathered}
$$

where the value of the angle $\varphi$ is fixed as $\pi / 2, \phi_{i}(r, \theta, t)$ are velocity potentials for the two fluids with $i$ denoting $h$ or $l$, and the interface perturbation $s(\theta, t)$ corresponds to $\eta(x, t)$ referring to the interface of two flat-substrate fluids in Cartesian geometry. The Laplace equation (2a) comes from the incompressibility condition in spherical geometry, Equations (2b) and (2c) represent the kinematic boundary conditions in spherical geometry (i.e., the normal velocity continuous condition on the interface) that a fluid particle initially situated at the material interface remains on the interface afterwards, and the Bernoulli equation (2d) comes from the dynamic boundary condition where the pressure continues across the interface. ${ }^{13}$

We consider an initial perturbation in the form

$$
r=s(\theta, t=0)=r_{0}+\varepsilon \cos (\kappa \theta),
$$

where $r_{0}$ is a positive constant, mode number $\kappa=2 \pi r_{0} / \lambda$ should be an integral and $\varepsilon \ll \lambda$. This simple perturbation, according to Eq. (5) in Ref. 14, is easy to investigate BP effect against planar RTI under the conditions that the perturbation distributes in just one coordinate and the character length is selected as wavelength, and the initial amplitude of the perturbation is far less than wavelength. This spherical perturbation is with the same mode number for variable polar angle $\varphi$. Because of the small amplitude perturbation in the spherical interface, this perturbed interface is prone to RTI, and higher harmonics (i.e., the second harmonic, the third harmonic, and so on) will subsequently be generated by the nonlinear process. ${ }^{13}$ Hence, the $s(\theta, t) \phi_{l}(r, \theta, t)$ and $\phi_{h}(r, \theta, t)$ can be expanded into a power series in $\hat{\varepsilon}$ as

$$
\begin{gathered}
s(\theta, t)=r_{0} \zeta(t)+\sum_{n=1}^{N} s^{(n)}(\theta, t)+O\left(\hat{\varepsilon}^{N+1}\right), \\
\phi_{l}(r, \theta, t)=\sum_{n=1}^{N} \phi_{l}^{(n)}(r, \theta, t)+O\left(\hat{\varepsilon}^{N+1}\right), \\
\phi_{h}(r, \theta, t)=\sum_{n=1}^{N} \phi_{h}^{(n)}(r, \theta, t)+O\left(\hat{\varepsilon}^{N+1}\right),
\end{gathered}
$$


with

$$
\begin{gathered}
\zeta(t)=1+\sum_{n=1}^{\left[\frac{N}{2}\right]} \hat{\varepsilon}^{2 n} e^{2 n \check{\beta} t} \check{\alpha}_{2 n, 0}, \\
s^{(n)}(\theta, t)=\hat{\varepsilon}^{n} e^{n \check{\beta} t} \sum_{m=0}^{\left[\frac{n}{2}\right]-1} \check{\alpha}_{n, n-2 m} \cos (n-2 m) \kappa \theta+O\left(\hat{\varepsilon}^{N+1}\right), \\
\phi_{l}^{(n)}(r, \theta, t)=\hat{\varepsilon}^{n} e^{n \check{\beta} t} \sum_{m=0}^{\left[\frac{n}{2}\right]} \phi_{l, n, n-2 m^{\frac{1}{2}}\left(\sqrt{4 \kappa^{2}(n-2 m)^{2}+1}-1\right)} \\
\quad \times \cos (n-2 m) \kappa \theta+O\left(\hat{\varepsilon}^{N+1}\right), \\
\phi_{h}^{(n)}(r, \theta, t)=\hat{\varepsilon}^{n} e^{n \check{\beta} t} \sum_{m=0}^{\left[\frac{n}{2}\right]} \phi_{h, n, n-2 m} r^{-\frac{1}{2}\left(\sqrt{4 \kappa^{2}(n-2 m)^{2}+1}+1\right)} \\
\times \cos (n-2 m) \kappa \theta+O\left(\hat{\varepsilon}^{N+1}\right) .
\end{gathered}
$$

Here, the normalized small parameter $\hat{\varepsilon}=\varepsilon / \lambda \ll 1$, the $N$ is set as 3, Gauss's symbol $[n / 2]$ denotes a maximum integer that is less than or equal to $\mathrm{n} / 2$, and $\check{\beta}$ is the linear growth rate in spherical RTI. As mentioned above, we just plan to investigate the BP effect against planar RTI, so the perturbation for parameter $\varphi=\pi / 2$ is considered in this paper. However, this perturbation is distributed in the whole spherical interface. In order to keep the same mode number for different polar angles $\varphi$, the smaller the absolute value of the $\varphi$ is, the smaller the wavelength is. Therefore, the assumption $\hat{\varepsilon}=\varepsilon / \lambda \ll 1$ can be valid for larger $\varphi$. It should be noted that the limitation of the $\hat{\varepsilon}=\varepsilon / \lambda \ll 1$ is just for the initial amplitude of the perturbation on the spherical interface, different from that in the Mikaelian's work ${ }^{20}$ where the algebraic product of the evolution and mode number is far less than the radius of the spherical interface.

The time function $\zeta(t)$ is just the correction to the zeroth harmonic from the higher orders including the second order, the fourth order, and so on. That is to say, the first correction to the function $\zeta(t)$ comes from the second order, and then it should contain factor $\hat{\varepsilon}^{2}$. The $n$th correction from the $2 n$th order should be with $\hat{\varepsilon}^{2 n}$. As a result, the series in $\zeta(t)$ proceeds in powers of $\hat{\varepsilon}^{2 n}$. This function determines whether the interface moves with time: the relation $\zeta(t) \equiv 1$ means that the position of the interface will keep resting; otherwise, it will move from the initial position $r(t=0)=r_{0}$. The functions $s^{(n)}(\theta, t)$ and $\phi_{l}^{(n)}(r, \theta, t)\left[\phi_{h}^{(n)}(r, \theta, t)\right]$ are, respectively, $n$ th-order perturbed interface and $n$ th-order perturbed velocity potential for the inner light [outer heavy] fluid of the interface. Regarding the $(n-2 m)$ th Fourier harmonic at the $n$ th-order, when $m=0, s_{n-2 m}^{(n)}=\hat{\varepsilon}^{n} e^{n \tilde{\beta} t} \check{\alpha}_{n, n-2 m}\left[\phi_{l, n-2 m}^{(n)}\right.$ $=\hat{\varepsilon}^{n} e^{n \check{\beta} t} \phi_{l, n, n-2 m} r^{\left(\sqrt{4 \kappa^{2}(n-2 m)^{2}+1}-1\right) / 2} \quad$ or $\quad \phi_{h, n-2 m}^{(n)}=\hat{\varepsilon}^{n} e^{n \check{\beta} t}$ $\left.\phi_{h, n, n-2 m} r^{-\left(\sqrt{4 \kappa^{2}(n-2 m)^{2}+1}+1\right) / 2}\right]$ is a generation coefficient of the perturbation interface [generation coefficient of the velocity potential for the light or heavy fluid]; while $m>0$, it is a correction coefficient of the $n$ th-order for the perturbation interface [a correction coefficient of the velocity potential for the inner fluid or outer fluid of the interface]. Note that the perturbation velocity potentials $\phi_{h}(r, \theta, t)$ and $\phi_{l}(r, \theta, t)$ have satisfied the Laplace equation (2a) and the boundary conditions $\left.\nabla \phi_{h}\right|_{r \rightarrow+\infty}=0$ and $\left.\nabla \phi_{l}\right|_{r=0}=0$. And the $\check{\alpha}_{1,1}=1$ according to the initial condition. The coupling factors in the amplitudes of the Fourier harmonic $\check{\alpha}_{n, n-2 m}$, $(n=2, \cdots, N$, and $m=0,1, \cdots,[n / 2])$ and $\check{\beta}$ are what we would like to determine. The above analysis includes that in Ref. 14.

According to the solving method used in Ref. 35, this nonlinear system can be solved order by order. The linear growth rate and coupling factors of the first four harmonics with corrections up to the third-order can be expressed as

$$
\check{\beta}=\kappa \sqrt{\frac{2 g}{r_{0}}} \sqrt{\frac{A\left(A+\sqrt{4 \kappa^{2}+1}\right)\left[A^{2}\left(4 \kappa^{2}+1\right)-1\right]}{-A^{4}\left(4 \kappa^{2}+1\right)+2 A^{2}\left(8 \kappa^{4}+4 \kappa^{2}+1\right)-4 \kappa^{2}-1}},
$$

$$
\begin{gathered}
\check{\alpha}_{2,0}=-\frac{1}{2 r_{0}^{2}}, \\
\check{\alpha}_{2,2}=-\frac{A\left(-K_{0}\right)+\sqrt{K_{0}}\left(A \sqrt{4 K_{0}-3}-2\right)+A+\sqrt{4 K_{0}-3}}{4\left(\sqrt{4 K_{0}-3}-\sqrt{K_{0}}\right) r_{0}},
\end{gathered}
$$

$\check{\alpha}_{3,1}=\frac{A^{2}\left(A^{2} K_{4}+2 A K_{5}+2 K_{3}\right)+2 K_{1}\left(4 A \kappa^{2}+A\right)+K_{2}\left(4 \kappa^{2}+1\right)}{64 K_{6} r_{0}^{2}\left(\sqrt{16 \kappa^{2}+1}+1\right)\left(-A^{2}+4 \kappa^{2}+1\right)}$,

$$
\check{\alpha}_{3,3}=\frac{3 A^{4} K_{8}+A^{3} K_{7}+A^{2} K_{11}+A K_{9}-2 K_{10}\left(4 \kappa^{2}+1\right)}{48 K_{12} K_{13} r_{0}^{2}\left(\sqrt{16 \kappa^{2}+1}+1\right)},
$$

where $K_{0}-K_{13}$ are attached in the Appendix.

Formula (6a) expresses that the linear growth rates in spherical and Cartesian geometries are of difference. Keeping Atwood number $A$, acceleration $g$ and mode number $\kappa$ invariable, the smaller the initial radius of the interface $r_{0}$ is, the larger the linear growth rate in the spherical geometry is. It is easy to find that when the critical Atwood number $A_{c}=1 /\left(4 \kappa^{2}+1\right)$, the normalized growth rate $\breve{\beta}$ is zero, and when $A<A_{c}$, the $\check{\beta}$ is an imaginary number. These show that when $A \leq A_{c}$, the spherical RTI will vanish, and only when $A>A_{c}$, it will happen. For example, $A_{c}=1 / 5$ if $\kappa=1$ and $A_{c}=1 / 37$ if $\kappa=3$, and so on. As the mode number $\kappa$ increases, the critical Atwood number $A_{c}$ will approach zero. This trend of the critical Atwood number with mode number is also predicted in Ref. 20. However, for the specific mode number, the critical Atwood number in this paper is much less than theirs. In addition, expressions (6c)-(6e) demonstrate that coupling factors are influenced by not only $A$ but also $\kappa$ and $r_{0}$

If the constant $\lambda$ is considered in both the spherical and Cartesian geometries [i.e., $\kappa / r_{0}=k$ ], and $\hat{r}_{0}$ is large [i.e., $\hat{r}_{0} \rightarrow+\infty$ ], the interface constructed by the above results of the spherical RTI will be reduced to that corresponding to the planar one. That is, the first four harmonics in spherical RTI will be simplified to those [see Eqs. (1a)-(1c)] in planar 

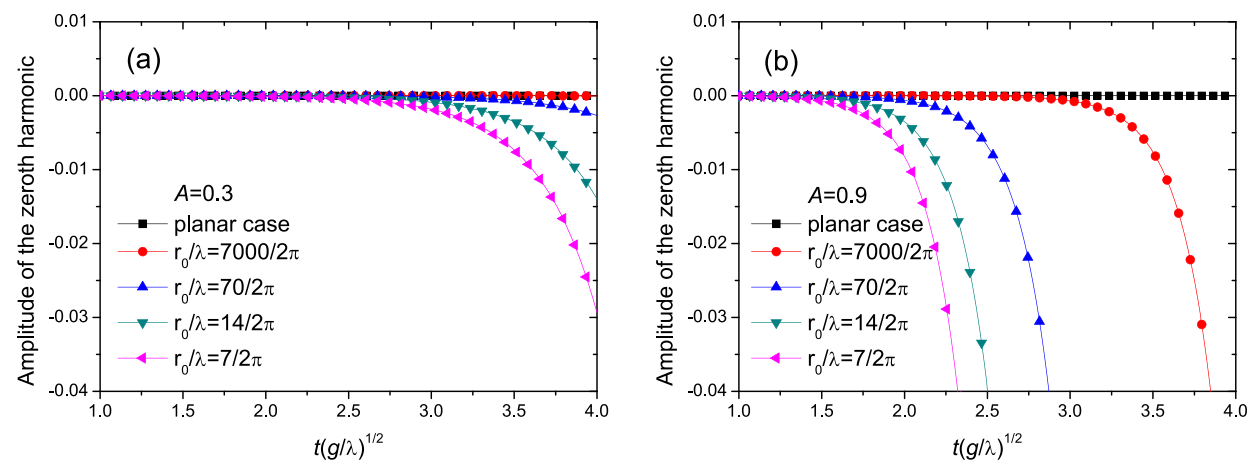

FIG. 1. The amplitude evolution of the zeroth harmonic, $r_{0} \check{\alpha}_{2,0} \eta_{L s}^{2} / \lambda$, versus time $t \sqrt{g / \lambda}$ for Atwood numbers $A=0.3$ (a) and $A=0.9$ (b). The initial amplitude is $\varepsilon / \lambda=1 / 1000$
RTI. In this configuration, it should be noted that the feedback to the zeroth harmonic from the second-order will vanish away, which can be easily confirmed in Eq. (6b). Note that the generation of $\check{\alpha}_{2,0}$ is an essential character completely different from the result in Cartesian geometry where $\alpha_{2,0}=0$.

Accordingly, the interface function at the framework of the third-order theory in the spherical RTI takes the form $r \doteq s_{0}+\sum_{n=1}^{3} s_{n} \cos (n \kappa \theta)$, where the $s_{0}$ and the amplitude of the $n$th harmonic, $s_{n}$, are

$$
\begin{gathered}
s_{0}=\zeta_{0}, \\
\zeta=1+\check{\alpha}_{2,0} \eta_{L s}^{2}, \\
s_{1}=\eta_{L s}\left(1+\check{\alpha}_{3,1} \eta_{L s}^{2}\right), \\
s_{2}=\check{\alpha}_{2,2} \eta_{L s}^{2}, \\
s_{3}=\check{\alpha}_{3,3} \eta_{L s}^{3},
\end{gathered}
$$

where $\eta_{L s}=\varepsilon e^{\check{\beta} t}$ is the linear growth amplitude of the fundamental mode in the spherical geometry. It should be pointed out that just the amplitude of the fundamental mode is corrected by the third harmonics, but the second and the third harmonics are not. As stated just now, an essential character different from the Cartesian case is that the zeroth harmonic appears in spherical RTI [see Equation (7b)]. This means that the position of the interface will be changed from the initial unperturbed interface $r=r_{0}$ into $r=\zeta(t) r_{0}$ with the evolution of the perturbation. The zeroth harmonic has an effect of reducing the radius of the spherical interface. Substitute Eqs. (7b) and (6b) to Eq. (7a), one finds that the second-order negative feedback to the zeroth harmonic [i.e., the initial zeroth interface $r=r_{0}$ ] decreases the radius of the spherical interface. However, this phenomenon of the second-order negative feedback to the zeroth harmonic does not appear for the planar RTI. That is to say, for the planar RTI, the zeroth interface keeps invariable all the time, but for the spherical RTI, it moves toward the center of the spherical space.

\section{HARMONIC EVOLUTION}

Because of the nonlinearity, high harmonics will be generated in quick succession and the initial interface developing in linear growth will be reduced. The interface includes two sections: the initial unperturbed interface known as the zeroth harmonic and perturbed interface. Within the thirdorder theory, the zeroth harmonic has a second-order correction, the fundamental mode (the first harmonic) does the third-order one, and the second and third harmonics have no feedback from higher orders. For unity, we use the characteristic quantities $\lambda$ and $g$ to normalize the initial radius and the time. The evolution of the amplitude of these four harmonics is considered in this paper. Figures 1-4 show the evolution of the zeroth, first, second, and third harmonics and Fig. 6 shows the third-order feedback to the fundamental mode. In order to better understand the spherical effect compared with the planar case, at Atwood number $A=0.3$ or $A=0.9$, we take the initial radius of the unperturbed interface as $\hat{r}_{0}=7 / 2 \pi, 14 / 2 \pi, 70 / 2 \pi, 7000 / 2 \pi$, and infinity (i.e., the amplitudes of the first, second and third harmonic expressed by Eqs. (1a)-(1c), and the amplitude of the zeroth harmonic known as nothing), respectively. The initial amplitude of the perturbed interface is set as $\hat{\varepsilon}=1 / 1000$.

Figure 1 shows that for the case of large $\hat{r}_{0}$, the amplitude of the zeroth harmonic tends to zero (i.e., the planar result). With the $\hat{r}_{0}$ decreasing, the amplitude of the zeroth harmonic quickly increases. That's to say, the spherical effect inspires the appearance of the zeroth harmonic, which
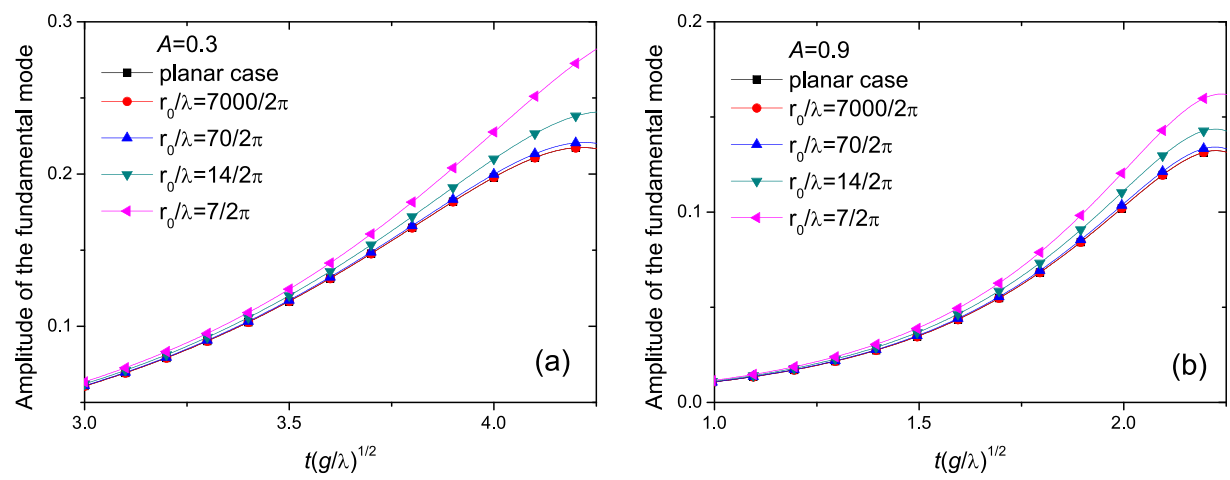

FIG. 2. The amplitude evolution of the first harmonic, $s_{1} / \lambda$, versus time $t \sqrt{g / \lambda}$ for Atwood numbers $A=0.3$ (a) and $A=0.9$ (b). The initial amplitude is $\varepsilon / \lambda=1 / 1000$. 


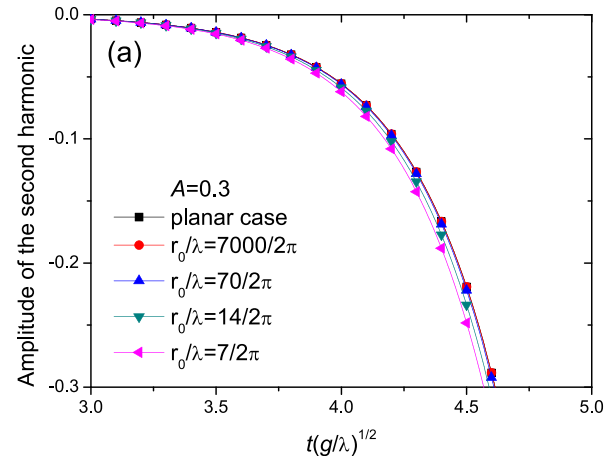

vanishes in the planar RTI. The negative amplitude of the zeroth harmonic indicates that the unperturbed interface starts moving towards to the spherical center. The unperturbed interface in planar RTI, nevertheless, keeps rest. Accordingly, the phenomenon that the unperturbed interface evolves to the spherical center is an innate character in spherical RTI. This character is more remarkable for the larger Atwood number.

Figure 2 denotes that the fundamental mode has the same trend as the planar result. With the time, the amplitude of the fundamental mode increases to its maximum value (i.e., saturation value) firstly, and then decreases. For the smaller Atwood number $(A=0.3)$, the smaller the initial radius of the interface is, the later the amplitude reaches its saturation value [see Fig. 2(a)]; however, for the larger Atwood number $(A=0.9)$, the amplitude saturates at almost the same time [see Fig. 2(b)]. In addition, especially for the smaller Atwood number, the smaller the radius is, the larger the amplitude is. Certainly, the phenomenon of the decreasing amplitude of the fundamental mode is off normal, so our third-order weakly nonlinear theory cannot predict the evolution of the fundamental mode any longer. That is to say, our theory is valid for the normalized time $t(g / \lambda)^{1 / 2}<4.2$ at $A=0.3$ and $t(g / \lambda)^{1 / 2}<2.5$ at $A=0.9$. This abnormal phenomenon appears when the third-order negative feedback to the fundamental mode is stronger than the linear growth of the fundamental mode. Therefore, if one wants to predict the amplitude evolution of the fundamental mode for longer time, the consideration of higher-order perturbations is needed.

The amplitude of the second harmonic in Fig. 3 is found to grow negatively for the different initial radius of the interface. When the normalized radius $\hat{r}_{0}$ tends to infinity, the amplitude tends to be the result of the planar RTI. With the decreasing $\hat{r}_{0}$, the amplitude grows fast, especially for the larger Atwood number.
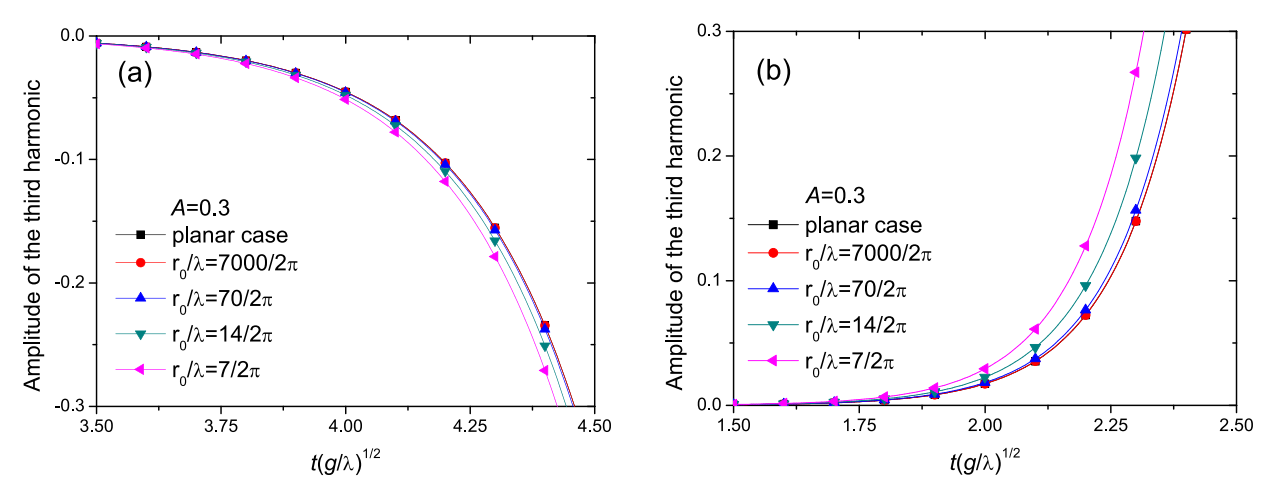

FIG. 3. The amplitude evolution of the second harmonic, $s_{2} / \lambda$, versus time $t \sqrt{g / \lambda}$ for Atwood numbers $A=0.3$ (a) and $A=0.9$ (b). The initial amplitude is $\varepsilon / \lambda=1 / 1000$.
In Fig. 4 , one finds that for $A=0.3$, the amplitude of the third harmonic grows negatively, while for $A=0.9$, it does positively. Whatever its positive or negative growth, the smaller the radius is, the faster it grows. This is more distinct for the larger Atwood number.

Two puzzling questions in the above discussion need us to further research. One is that the spherical effect makes the zeroth, second, and third harmonics grow rapidly, especially for the larger Atwood number, while for the fundamental mode, the spherical effect makes it grow faster for the smaller Atwood number than the larger one. The other is that the amplitude of the third harmonic has the positive or negative growth.

In this paper, we just consider the harmonic to the third order. Hence, the zeroth harmonic and the first one are separately corrected by the second order and third order, and the second and third harmonics are not. From Fig. 5, one can see that for different initial radii, the linear amplitude of the fundamental mode is strengthened by the spherical effect, especially for the larger Atwood number. Figure 6 shows that the smaller the radius is, the weaker and the later the feedback to the fundamental mode is, especially for the smaller Atwood number. This can help us better understand the first question mentioned above. The amplitude of the third harmonic grows either positively or negatively, depending on the factor $\check{\alpha}_{3,3}$. When the value of the $\check{\alpha}_{3,3}>0\left(\check{\alpha}_{3,3}<0\right)$, the amplitude grows positively (negatively). When $\check{\alpha}_{3,3}=0$, it vanishes. From Fig. 7, one finds that the factor $\check{\alpha}_{3,3}$ changes with the Atwood number and the initial radius. It is obvious that the critical Atwood number is 0.5 . At $A<0.5$, the factor $\check{\alpha}_{3,3}<0$ and for $A>0.5$, the factor $\check{\alpha}_{3,3}>0$. This means that the amplitude of the third harmonic grows positively at $A>0.5$ or negatively at $A<0.5$. When $A=0.5$, the third harmonic will vanish.

FIG. 4. The amplitude evolution of the third harmonic, $s_{3} / \lambda$, versus time $t \sqrt{g / \lambda}$ for Atwood numbers $A=0.3$ (a) and $A=0.9$ (b). The initial amplitude is $\varepsilon / \lambda=1 / 1000$. 

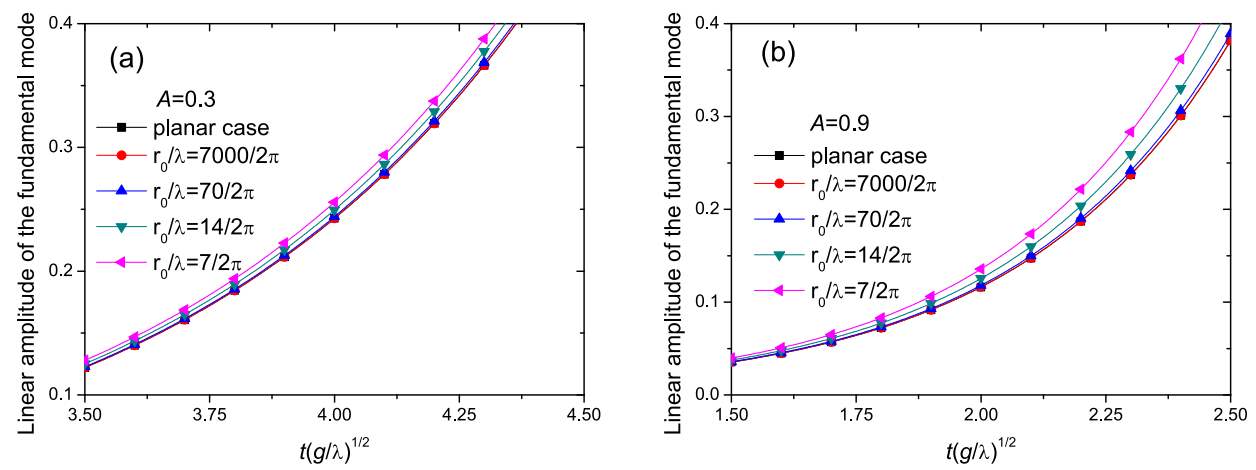

FIG. 5. The linear amplitude of the fundamental mode, $\eta_{L s} / \lambda$, versus time $t \sqrt{g / \lambda}$ for Atwood numbers $A=0.3$ (a) and $A=0.9$ (b). The initial amplitude is $\varepsilon / \lambda=1 / 1000$.
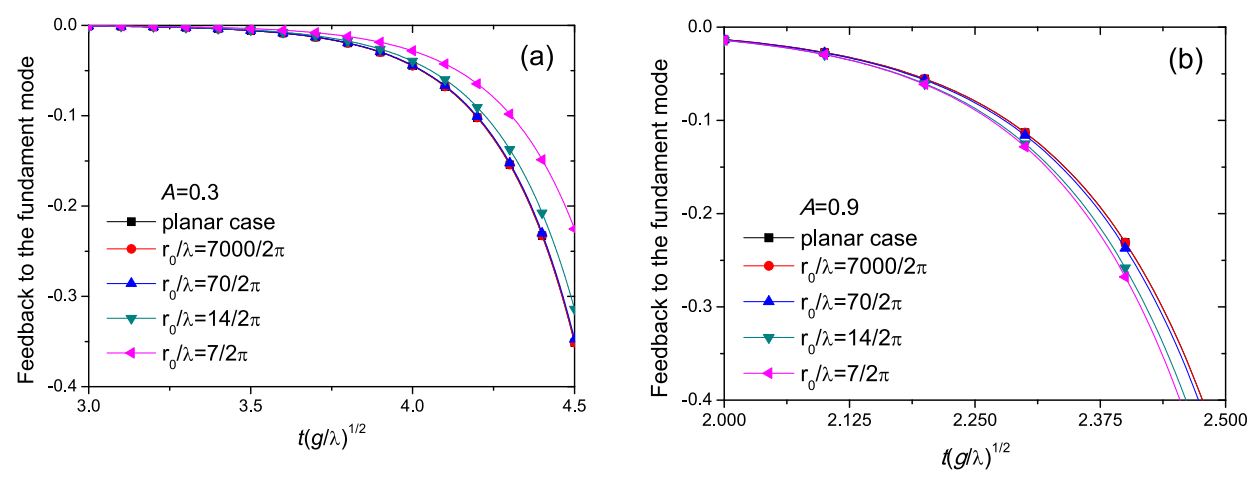

FIG. 6. The amplitude evolution of the third-order feedback to the fundamental mode, $\check{\alpha}_{3,1} \eta_{L s}^{3} / \lambda$, versus time $t \sqrt{g / \lambda}$ for Atwood numbers $A=0.3$ (a) and $A=0.9$ (b). The initial amplitude is $\varepsilon / \lambda=1 / 1000$.

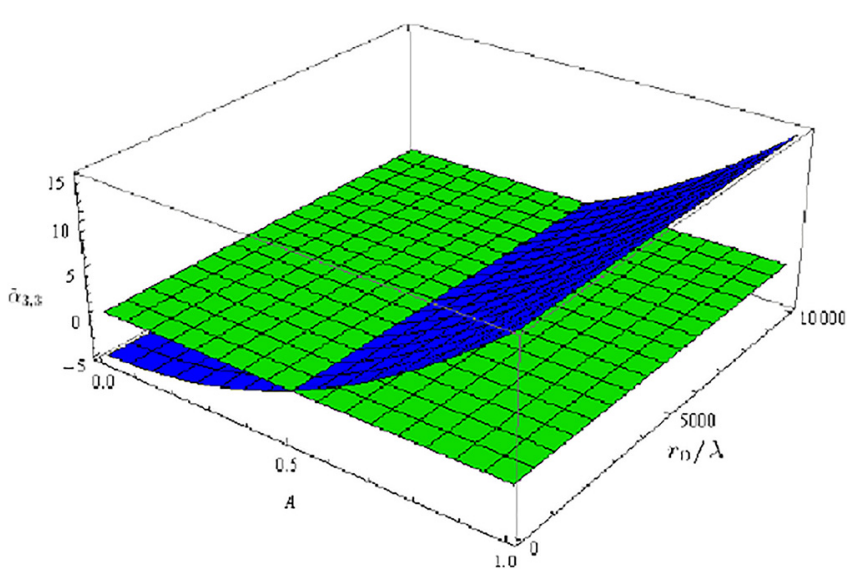

FIG. 7. The factor $\check{\alpha}_{3,3}$ in the amplitude of the third harmonic versus Atwood number and the initial radius (curved surface). The value of the zero is indicated by the planar surface.

\section{CONCLUSION}

In this investigation, we have used the method of the small parameter expansion with nonlinear corrections up to the third order to analytically explore the amplitude evolution of the first four harmonics in the classical RTI (irrotational, incompressible, and inviscid fluids) with a discontinuous profile in spherical geometry for arbitrary Atwood numbers. Take the same initial wavelength and large initial radius, then our spherical results will tend to those in planar RTI where the zeroth harmonic does not appear.

Unlike the planar RTI, the second-order feedback to the zeroth harmonic always grows negatively for the arbitrary Atwood number and initial radius. This will lead to the initial unperturbed interface to move towards to the center of the spherical system. Especially for the large Atwood number, the smaller the initial radius is, the faster the initial unperturbed interface moves. In another word, for the large Atwood number, the spherical effect has a great influence on the interface.

The spherical effect strengthens the amplitude growth of linear harmonics, which are not corrected by the higher order, e.g., the linear amplitude of the fundament mode $\eta L c$, the second and third harmonics for the arbitrary Atwood number. The larger Atwood number is, the faster they grow. For the fundamental mode corrected by the third order, the spherical effect still strengthens its growth. However, the smaller Atwood number is, the faster the amplitude of the fundament mode grows. This is due to the feedback quantity from the third order. Although the third order gives the fundamental mode a negative correction, the stronger the spherical effect is (that is, the smaller the initial radius), the weaker the feedback is. Thus, for the smaller Atwood number, the spherical effect strengthens the linear growth of the fundamental mode, and weakens the feedback to the fundamental mode from the third order.

The third harmonic grows positively or negatively, depending on the factor $\check{\alpha}_{3,3}$, which is a function of coupling the initial radius and Atwood number. The $\check{\alpha}_{3,3}>0$ $\left(\check{\alpha}_{3,3}<0\right)$ corresponds to the positive (negative) growth. However, we find that for the arbitrary initial radius, the Atwood number controls its growth. When the $A<0.5$ $(A>0.5)$, it grows negatively (positively).

\section{SUPPLEMENTARY MATERIAL}

See supplementary material for factors of the mode number. 


\section{ACKNOWLEDGMENTS}

All the authors would like to thank the anonymous referee for suggestions that have improved the paper. This work was supported by the National Natural Science Foundation of China (Grant Nos. 11472278 and 11372330), the National Key Research and Development Program of China (Grant No. 2016YFA0401200), the Scientific Research Foundation of Mianyang Normal University (Grant No. QD2014A009), and the National High-Tech ICF Committee.

\section{APPENDIX: FACTORS OF THE MODE NUMBER $\mathrm{K}_{0}-\mathrm{K}_{13}$}

The $K_{0}-K_{13}$ in coupling factors of the first four harmonics are

$$
K_{0}=4 \kappa^{2}+1,
$$

$$
\begin{aligned}
& K_{1}=576 \kappa^{4}+\left(-40 \sqrt{16 \kappa^{2}+1} \sqrt{4 \kappa^{2}+1}-40 \sqrt{4 \kappa^{2}+1}+36 \sqrt{16 \kappa^{2}+1}+724\right) \kappa^{2}-43\left(\sqrt{4 \kappa^{2}+1}-1\right)\left(\sqrt{16 \kappa^{2}+1}+1\right), \\
& K_{2}=\kappa^{4}\left(128 \sqrt{4 \kappa^{2}+1}-80 \sqrt{16 \kappa^{2}+1}-80\right)+\kappa^{2}\left(8 \sqrt{16 \kappa^{2}+1} \sqrt{4 \kappa^{2}+1}+1064 \sqrt{4 \kappa^{2}+1}-360 \sqrt{16 \kappa^{2}+1}-360\right) \\
&+ 66 \sqrt{16 \kappa^{2}+1} \sqrt{4 \kappa^{2}+1}+66 \sqrt{4 \kappa^{2}+1}-85 \sqrt{16 \kappa^{2}+1}-85, \\
& K_{3}=-96 \kappa^{6}\left(8 \sqrt{4 \kappa^{2}+1}-3 \sqrt{16 \kappa^{2}+1}-3\right)+8 \kappa^{4}\left(-6 \sqrt{16 \kappa^{2}+1} \sqrt{4 \kappa^{2}+1}+10 \sqrt{4 \kappa^{2}+1}+9 \sqrt{16 \kappa^{2}+1}+9\right) \\
&+8 \kappa^{2}\left(\sqrt{16 \kappa^{2}+1} \sqrt{4 \kappa^{2}+1}-45 \sqrt{4 \kappa^{2}+1}+21 \sqrt{16 \kappa^{2}+1}+21\right)-\left(23 \sqrt{4 \kappa^{2}+1}-42\right)\left(\sqrt{16 \kappa^{2}+1}+1\right), \\
& K_{4}=-48 \kappa^{4}\left(8 \sqrt{4 \kappa^{2}+1}-3 \sqrt{16 \kappa^{2}+1}-3\right)-4 \kappa^{2}\left(6 \sqrt{16 \kappa^{2}+1} \sqrt{4 \kappa^{2}+1}+86 \sqrt{4 \kappa^{2}+1}-25 \sqrt{16 \kappa^{2}+1}-25\right) \\
&-\left(20 \sqrt{4 \kappa^{2}+1}-1\right)\left(\sqrt{16 \kappa^{2}+1}+1\right),
\end{aligned}
$$

$K_{5}=\kappa^{4}\left(144 \sqrt{16 \kappa^{2}+1} \sqrt{4 \kappa^{2}+1}+144 \sqrt{4 \kappa^{2}+1}-96 \sqrt{16 \kappa^{2}+1}-1312\right)+\kappa^{2}\left(80 \sqrt{16 \kappa^{2}+1} \sqrt{4 \kappa^{2}+1}+80 \sqrt{4 \kappa^{2}+1}\right.$$$
\left.-76 \sqrt{16 \kappa^{2}+1}-764\right)-1536 \kappa^{6}+43 \sqrt{4 \kappa^{2}+1}+43 \sqrt{4 \kappa^{2}+1} \sqrt{16 \kappa^{2}+1}-43 \sqrt{16 \kappa^{2}+1}-43 \text {, }
$$

$$
K_{6}=-A \sqrt{4 \kappa^{2}+1}+A \sqrt{16 \kappa^{2}+1}-4 \kappa^{2}+\sqrt{4 \kappa^{2}+1} \sqrt{16 \kappa^{2}+1}-1,
$$

$$
K_{7}=12288 \kappa^{6}-96 \kappa^{4}\left(11 \sqrt{16 \kappa^{2}+1} \sqrt{4 \kappa^{2}+1}+11 \sqrt{4 \kappa^{2}+1}-8 \sqrt{16 \kappa^{2}+1}+3 \sqrt{16 \kappa^{2}+1} \sqrt{36 \kappa^{2}+1}\right)-96 \kappa^{4}
$$$$
\times\left(3 \sqrt{36 \kappa^{2}+1}-58\right)-4 \kappa^{2}\left(75 \sqrt{16 \kappa^{2}+1} \sqrt{4 \kappa^{2}+1}+60 \sqrt{36 \kappa^{2}+1} \sqrt{4 \kappa^{2}+1}\right)-4 \kappa^{2}\left(75 \sqrt{4 \kappa^{2}+1}\right.
$$$$
\left.-75 \sqrt{16 \kappa^{2}+1}+9 \sqrt{16 \kappa^{2}+1} \sqrt{36 \kappa^{2}+1}+9 \sqrt{36 \kappa^{2}+1}-119\right) \text {, }
$$

$$
\begin{aligned}
K_{8}= & 16 \kappa^{4}\left(32 \sqrt{4 \kappa^{2}+1}-11 \sqrt{16 \kappa^{2}+1}-11\right)-4 \kappa^{2}\left(-8 \sqrt{16 \kappa^{2}+1} \sqrt{4 \kappa^{2}+1}\right)-4 \kappa^{2}\left(3 \sqrt{16 \kappa^{2}+1} \sqrt{36 \kappa^{2}+1}\right. \\
& \left.\times \sqrt{4 \kappa^{2}+1}+3 \sqrt{36 \kappa^{2}+1} \sqrt{4 \kappa^{2}+1}-12 \sqrt{4 \kappa^{2}+1}\right)-4 \kappa^{2}\left(6 \sqrt{16 \kappa^{2}+1}+4 \sqrt{36 \kappa^{2}+1}+6\right) \\
& -\left(\sqrt{16 \kappa^{2}+1}+1\right)\left(\sqrt{36 \kappa^{2}+1} \sqrt{4 \kappa^{2}+1}-\sqrt{4 \kappa^{2}+1}+\sqrt{36 \kappa^{2}+1}+1\right),
\end{aligned}
$$

$$
\begin{aligned}
K_{9}= & -4608 \kappa^{6}-\left(\sqrt{16 \kappa^{2}+1}+1\right)\left(25 \sqrt{36 \kappa^{2}+1} \sqrt{4 \kappa^{2}+1}+41 \sqrt{4 \kappa^{2}+1}-37 \sqrt{36 \kappa^{2}+1}-29\right)+32 \kappa^{4}\left(9 \sqrt{16 \kappa^{2}+1}\right. \\
& \left.\times \sqrt{4 \kappa^{2}+1}-36 \sqrt{36 \kappa^{2}+1} \sqrt{4 \kappa^{2}+1}+9 \sqrt{4 \kappa^{2}+1}-9 \sqrt{16 \kappa^{2}+1}\right)+32 \kappa^{4}\left(21 \sqrt{16 \kappa^{2}+1} \sqrt{36 \kappa^{2}+1}\right. \\
+ & \left.21 \sqrt{36 \kappa^{2}+1}+13\right)-4 \kappa^{2}\left(23 \sqrt{16 \kappa^{2}+1} \sqrt{4 \kappa^{2}+1}+18 \sqrt{16 \kappa^{2}+1} \sqrt{36 \kappa^{2}+1} \sqrt{4 \kappa^{2}+1}+118 \sqrt{36 \kappa^{2}+1}\right. \\
& \left.\times \sqrt{4 \kappa^{2}+1}\right)-4 \kappa^{2}\left(23 \sqrt{4 \kappa^{2}+1}-11 \sqrt{16 \kappa^{2}+1}-79 \sqrt{16 \kappa^{2}+1} \sqrt{36 \kappa^{2}+1}-79 \sqrt{36 \kappa^{2}+1}-127\right), \\
K_{10}= & 12 \kappa^{4}\left(52 \sqrt{4 \kappa^{2}+1}-19 \sqrt{16 \kappa^{2}+1}+12 \sqrt{36 \kappa^{2}+1}-19\right)-\kappa^{2}\left(-39 \sqrt{16 \kappa^{2}+1} \sqrt{4 \kappa^{2}+1}+27 \sqrt{16 \kappa^{2}+1}\right. \\
& \left.\times \sqrt{36 \kappa^{2}+1} \sqrt{4 \kappa^{2}+1}+27 \sqrt{36 \kappa^{2}+1} \sqrt{4 \kappa^{2}+1}\right)-\kappa^{2}\left(57 \sqrt{4 \kappa^{2}+1}+9 \sqrt{16 \kappa^{2}+1}-9 \sqrt{16 \kappa^{2}+1} \sqrt{36 \kappa^{2}+1}\right. \\
& \left.-73 \sqrt{36 \kappa^{2}+1}+9\right)-\left(\sqrt{16 \kappa^{2}+1}+1\right)\left(7 \sqrt{36 \kappa^{2}+1} \sqrt{4 \kappa^{2}+1}+6 \sqrt{4 \kappa^{2}+1}-4 \sqrt{36 \kappa^{2}+1}-12\right),
\end{aligned}
$$




$$
\begin{aligned}
K_{11}= & \kappa^{6}\left(6144 \sqrt{4 \kappa^{2}+1}-2112 \sqrt{16 \kappa^{2}+1}-2112\right)+\kappa^{4}\left(384 \sqrt{16 \kappa^{2}+1} \sqrt{4 \kappa^{2}+1}-144 \sqrt{16 \kappa^{2}+1} \sqrt{36 \kappa^{2}+1}\right. \\
& \left.\times \sqrt{4 \kappa^{2}+1}\right)+\kappa^{4}\left(-144 \sqrt{36 \kappa^{2}+1} \sqrt{4 \kappa^{2}+1}+3552 \sqrt{4 \kappa^{2}+1}-1608 \sqrt{16 \kappa^{2}+1}-1632 \sqrt{36 \kappa^{2}+1}-1608\right) \\
& +\kappa^{2}\left(198 \sqrt{16 \kappa^{2}+1} \sqrt{4 \kappa^{2}+1}+126 \sqrt{16 \kappa^{2}+1} \sqrt{36 \kappa^{2}+1} \sqrt{4 \kappa^{2}+1}+126 \sqrt{36 \kappa^{2}+1} \sqrt{4 \kappa^{2}+1}\right) \\
& +\kappa^{2}\left(598 \sqrt{4 \kappa^{2}+1}-322 \sqrt{16 \kappa^{2}+1}-102 \sqrt{16 \kappa^{2}+1} \sqrt{36 \kappa^{2}+1}-566 \sqrt{36 \kappa^{2}+1}-322\right)+25 \sqrt{16 \kappa^{2}+1} \\
& \times \sqrt{4 \kappa^{2}+1}+29 \sqrt{16 \kappa^{2}+1} \sqrt{36 \kappa^{2}+1} \sqrt{4 \kappa^{2}+1}+29 \sqrt{36 \kappa^{2}+1} \sqrt{4 \kappa^{2}+1}+25 \sqrt{4 \kappa^{2}+1}-13 \sqrt{16 \kappa^{2}+1} \\
& -29 \sqrt{16 \kappa^{2}+1} \sqrt{36 \kappa^{2}+1}-29 \sqrt{36 \kappa^{2}+1}-13
\end{aligned}
$$

$$
\begin{aligned}
K_{12}= & A\left(\sqrt{4 \kappa^{2}+1}-\sqrt{36 \kappa^{2}+1}\right)+4 \kappa^{2} \\
& -\sqrt{4 \kappa^{2}+1} \sqrt{36 \kappa^{2}+1}+1, \\
K_{13}= & A\left(\sqrt{16 \kappa^{2}+1}-\sqrt{4 \kappa^{2}+1}\right)-4 \kappa^{2} \\
& +\sqrt{4 \kappa^{2}+1} \sqrt{16 \kappa^{2}+1}-1 .
\end{aligned}
$$

${ }^{1}$ J. Nuckolls, L. Wood, A. Thiessen, and G. Zimmerman, Nature 239, 139 (1972).

${ }^{2}$ J. D. Lindl, Phys. Plasmas 2, 3933 (1995).

${ }^{3}$ V. A. Smalyuk, V. N. Goncharov, T. R. Boehly, J. P. Knauer, D. D. Meyerhofer, and T. C. Sangster, Phys. Plasmas 11, 5038 (2004).

${ }^{4}$ D. Layzer, Astrophys. J. 122, 1 (1955).

${ }^{5}$ J. W. Jacobs and I. Catton, J. Fluid Mech. 187, 329 (1988).

${ }^{6}$ S. W. Haan, Phys. Fluids B 3, 2349 (1991).

${ }^{7}$ M. Berning and A. M. Rubenchik, Phys. Fluids 10, 1564 (1998).

${ }^{8}$ S. Hasegawa and K. Nishihara, Phys. Plasmas 2(12), 4606 (1995).

${ }^{9}$ J. Sanz, J. Ramìrez, R. Ramis, R. Betti, and R. P. J. Town, Phys. Rev. Lett. 89, 195002 (2002).

${ }^{10}$ T. Ikegawa and K. Nishihara, Phys. Rev. Lett. 89, 115001 (2002).

${ }^{11}$ J. Garnier and L. Masse, Phys. Plasmas 12, 062707 (2005).

${ }^{12}$ W. H. Liu, L. F. Wang, W. H. Ye, and X. T. He, Phys. Plasmas 19, 042705 (2012).

${ }^{13}$ W. H. Liu, W. F. Ma, and X. L. Wang, Chin. Phys. B 24, 015202 (2015).

${ }^{14}$ W. H. Liu, Y. L. Chen, C. P. Ye, and X. L. Li, Phys. Plasmas 22, 112112 (2015).
${ }^{15}$ B. A. Remington, S. W. Haan, S. G. Glendinning, J. D. Kilkenny, D. H. Munro, and R. J. Wallace, Phys. Fluids B 4(4), 967 (1992).

${ }^{16}$ B. A. Remington, S. V. Weber, M. M. Marinak, S. W. Haan, J. D. Kilkenny, R. J. Wallace, and G. Dimonte, Phys. Plasmas 2(1), 241 (1995).

${ }^{17}$ M. J. Dunning and S. W. Haan, Phys. Plasmas 2(5), 1669 (1995).

${ }^{18}$ L. F. Wang, W. H. Ye, Z. F. Fan, and Y. J. Li, Europhys. Lett. 90, 15001 (2010).

${ }^{19}$ L. F. Wang, W. H. Ye, and Y. J. Li, Phys. Plasmas 17, 052305 (2010).

${ }^{20}$ K. O. Mikaelian, Phys. Rev. A 42(6), 3400 (1990).

${ }^{21}$ Q. Zhang, Phys. Rev. Lett. 81(16), 3391 (1998).

${ }^{22}$ K. O. Mikaelian, Phys. Rev. E 67, 026319 (2003).

${ }^{23}$ V. N. Goncharov, Phys. Rev. Lett. 88(13), 134502 (2002).

${ }^{24}$ P. Ramaprabhu, G. Dimonte, Y. N. Young, A. C. Calder, and B. Fryxell, Phys. Rev. E 74, 066308 (2006).

${ }^{25}$ L. Rayleigh, Proc. London Math. Soc. 14, 170 (1883).

${ }^{26}$ G. Taylor, Proc. R. Soc. London, Ser. A 201, 192 (1950).

${ }^{27}$ G. Terronesa and M. D. Carrara, Phys. Fluids 27, 054105 (2015).

${ }^{28}$ G. I. Bell, Los Alamos National Laboratory, Report No. LA-1321 (1951).

${ }^{29}$ M. S. Plesset, J. Appl. Phys. 25, 96 (1954).

${ }^{30}$ P. Amendt, J. D. Colvin, J. D. Ramshaw, H. F. Robey, and O. L. Landen, Phys. Plasmas 10, 820 (2003).

${ }^{31}$ K. O. Mikaelian, Phys. Fluids 17, 094105 (2005).

${ }^{32} \mathrm{H}$. Yu and D. Livescu, Phys. Fluids 20, 104103 (2008).

${ }^{33}$ L. K. Forbes, ANZIAM J. 53, 87 (2011).

${ }^{34}$ W. W. Hsing, C. W. Barnes, J. B. Beck, N. M. Hoffman, D. Galmiche, A. Richard, J. Edwards, P. Graham, S. Rothman, and B. Thomas, Phys. Plasmas 4, 1832 (1997).

${ }^{35}$ W. H. Liu, C. P. Yu, W. H. Ye, L. F. Wang, and X. T. He, Phys. Plasmas 21, 062119 (2014). 